\title{
Nephron-Sparing Diagnosis and Management of Renal Keratinizing Desquamative Squamous Metaplasia
}

\author{
Michael Borofsky, B.A., ${ }^{1}$ Rajal B. Shah, M.D., ${ }^{2}$ and J. Stuart Wolf, Jr., M.D. ${ }^{1}$
}

\begin{abstract}
Background and Purpose: Keratinizing desquamative squamous metaplasia (KDSM) of the upper urinary tract is a rare condition for which there is no defined management plan. A condition historically treated with extirpative surgery, conservative management would be preferable, because this is almost certainly a benign condition. We report the favorable clinical course of two cases of renal KDSM diagnosed and managed with a nephron-sparing approach, relying on ureteroscopy and serial imaging.

Patients and Methods: Retrospective chart review was performed to obtain history, physical examination results, radiographic imaging, and diagnostic procedures.

Results: Both patients were referred to our institution for evaluation of complex cystic renal masses. Both reported passing flaky material in their urine. Flexible ureteroscopy revealed waxy sediment in the collecting system, which broke up easily with manipulation and proved to be squamous keratin debris on cytologic and histologic examination. In 1 patient, we obtained a percutaneous needle biopsy as well, which revealed benign keratinizing squamous epithelium. All findings were consistent with KDSM. Each patient has since been followed conservatively with CT. In 1 case, there has been slight growth of the mass but no worrisome changes after 42 months. In the other case, there were several new renal collecting system filling defects on CT 17 months after diagnosis. Another ureteroscopy revealed the same findings as the original, with the addition of keratin debris draining out of the lesion into the rest of the kidney.

Conclusions: Our two cases of KDSM confirm the feasibility of nephron-sparing management using a combination of diagnostic ureteroscopy and imaging surveillance. The duration of follow-up without adverse events suggests that the finding of renal KDSM is not necessarily an indication for extirpative surgery, and that conservative management is an appropriate option.
\end{abstract}

\section{Introduction}

K ERATINIZING DESQuAmATIVE SQuAmous METAPLASIA (KDSM) of the upper urinary tract is a rare condition for which there is no universally accepted management plan. The first account of KDSM of the urinary tract was by Rokitansky in 1861, and the first report of KDSM in the renal pelvis is credited to Ebstein in $1882 .{ }^{1}$ Despite a long history of reports, there is no consensus on the management of KDSM. Knowledge about the condition has been limited not only by lack of cases but also because it is often referred to by several different names, including cholesteatoma, leukoplakia, and hyperkeratosis.

There has been controversy about whether to manage renal KDSM as a benign or premalignant condition. Initially, it was considered a precancerous lesion, ${ }^{2}$ because squamous metaplasia is a precancerous finding in other tissues, such as the respiratory tract and cervix. ${ }^{3}$ Nonetheless, despite the similarities between squamous metaplasia of the upper urinary tract and of other tissues, the malignant potential of renal KDSM has never been demonstrated.

This has led many, including our group, to believe that a conservative strategy is appropriate. This approach is hindered by the tendency to perform extirpative surgery for the complex-appearing cystic masses that are characteristic of KDSM.

The purpose of this report is to define the favorable clinical course of two cases of KDSM managed in a nephronsparing manner with biopsy, diagnostic ureteroscopy, and radiographic surveillance. 
A

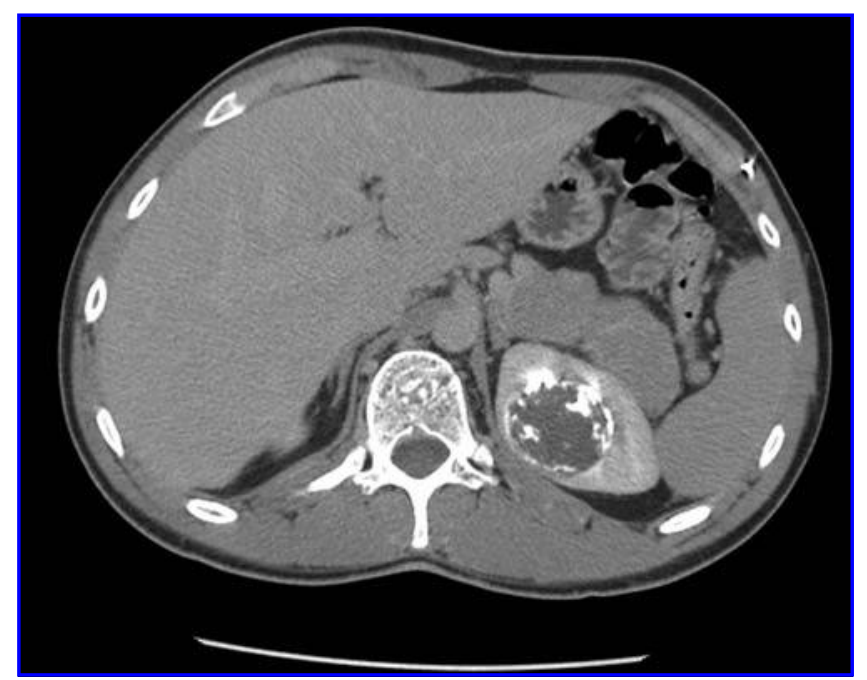

B

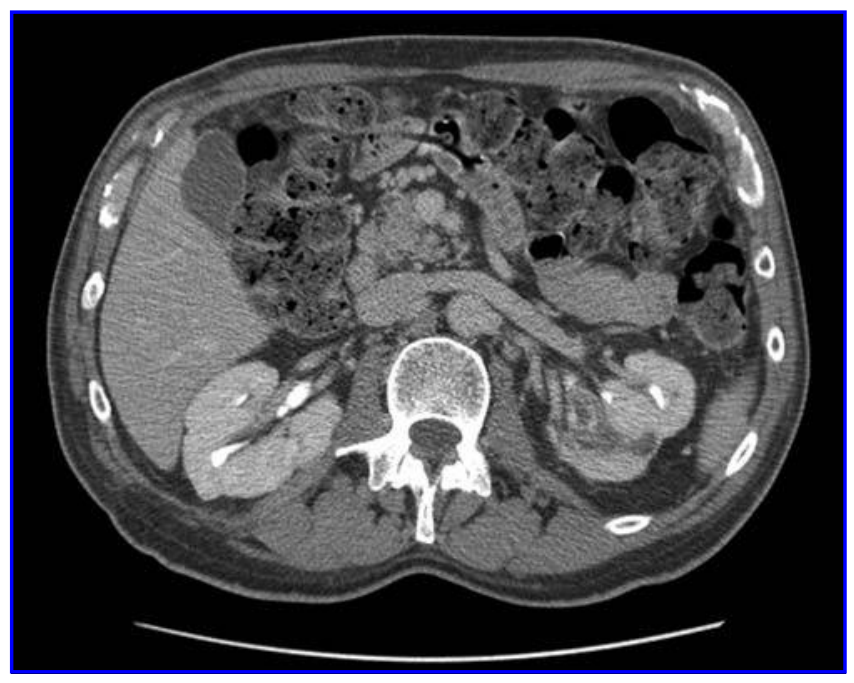

FIG. 1. (A) CT shows $5.0 \times 4.1 \mathrm{~cm}$ mixed attenuating mass in the upper pole of the left kidney, with bright enhancement in serpiginous areas after intravenous contrast material administration. (B) CT demonstrates $3.5 \times 2.9 \mathrm{~cm}$ loculated soft tissue mass in the upper pole of the left kidney with surrounding cortical atrophy, some enhancement, and no filling by urine.

\section{Patients and Methods}

The clinical courses of two patients referred to our institution with complex cystic renal masses that ultimately proved to be KDSM were retrospectively reviewed. History, physical examination findings, radiographic imaging, and diagnostic procedures were considered. All tests and procedures were performed on an outpatient basis. The patients were fully informed of the rare nature of their condition, that clear evidence to guide their management was lacking, and that further treatment up to and including nephroureterectomy might be necessary.

\section{Results}

Both patients referred to our institution with complex cystic renal masses were Caucasian men, 22 and 72 years of age. The younger patient had a history of nephrolithiasis and congenital heart disease, and the older man had a history of urinary tract infections and benign prostatic hyperplasia. The 22-year-old man presented with left flank pain, which prompted an abdominal CT that revealed a large mixed-density left renal mass. Subsequent retrograde pyelography showed contrast entering the mass.

The older patient also presented with left flank pain, prompting an abdominal CT that revealed left hydronephrosis and an enlarged prostate. He eventually underwent transurethral resection of the prostate and was referred to our institution after subsequent retrograde pyelography and ureteroscopy confirmed resolution of the hydronephrosis but the presence of a large filling defect in the upper pole of the left kidney, which contained debris but no definite mass. Both patients reported flaky material present in their urine.

CTs at our institution revealed similar findings in both patients. The younger man had a $5.0 \times 4.1 \mathrm{~cm}$ mixed attenuating mass in the upper pole of the left kidney, which enhanced brightly in serpiginous areas after intravenous contrast ma- terial administration (Fig. 1A). The older man had a $3.5 \times$ $2.9 \mathrm{~cm}$ loculated soft tissue mass in the upper pole of the left kidney with surrounding cortical atrophy. The lesion enhanced with intravenous contrast, but did not demonstrate the bright filling seen in the younger patient (Fig. 1B). There was a renal stone present as well.

Both patients underwent flexible ureteroscopy as the initial diagnostic evaluation at our institution. In both cases, findings included a large cystic structure emanating from the upper pole intrarenal collecting system. The interior of the structures contained a large amount of waxy gray to yellow sediment that fragmented easily with manipulation (Fig. 2).

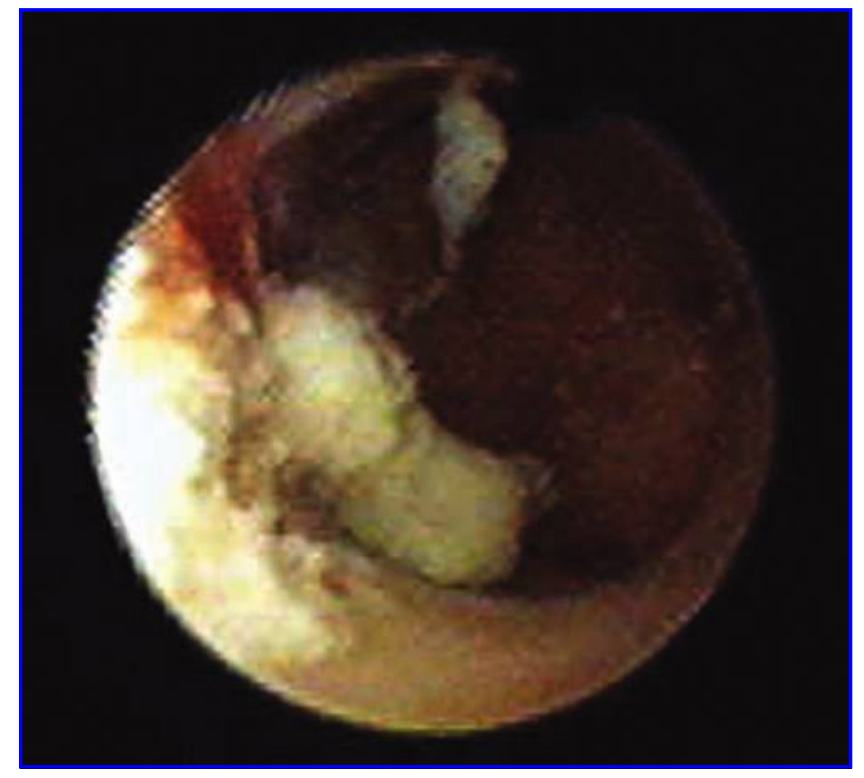

FIG. 2. Ureteroscopic view of waxy yellow debris in the collecting system. 
Washings after manipulation with baskets were sent for both cytologic and pathologic examination, which revealed squamous keratin debris without evidence of malignancy (Fig. $3 \mathrm{~A})$. In the older patient, to provide more definitive diagnosis, a percutaneous biopsy of the lesion was obtained, which revealed benign keratinizing squamous epithelium and debris of anucleated squamous cells (Fig. 3B).

Both patients have been followed with CT. The younger patient had one isolated episode of mild left flank that resolved. Over time, the mass has grown slightly, with his most recent $\mathrm{CT}, 42$ months after diagnosis, revealing an increase to $5.8 \times 4.5 \mathrm{~cm}$ (Fig. $4 \mathrm{~A}$ ). Because of the lack of any other worrisome change, surveillance continues.

In the older patient, CT 17 months after our initial evaluation revealed the mass to be unchanged, but now containing contrast material mixed with debris (Fig. 4B). Several new small filling defects were noted in the middle and lower calices and the renal pelvis (Fig. 4C, compared with previously normal collecting system from initial imaging in Fig. 4D). He reported an increasing volume of flaky material in the urine.

After discussing the possibility that these changes might represent an inflammatory response, but that malignancy had to be considered, we repeated ureteroscopy. This revealed inflamed mucosa in the bladder, ureter, and renal collecting system (Fig. 5) in addition to a large amount of debris. Irrigation of the bladder and collecting system resulted in a normal appearance aside from the inflammation, and results of cytologic evaluation from washings were negative for malignant cells. We conjectured that the changes seen on CT were a result of the first ureteroscopy, which enlarged the orifice of the lesion, allowing for the increased contrast material in the lesion on CT and also for greater passage of the keratin flakes through the urinary tract.

\section{Discussion}

While there has been controversy over the characterization, naming, and management of KDSM, the one consis- tency has been the histologic findings of a keratinizing layer of well-differentiated squamous epithelium without evidence of cellular atypia in the basal layer. ${ }^{1,3-9}$ The only neoplastic associations in the literature from the past 50 years are from Reece and Koontz, ${ }^{3}$ who reported two cases of concurrent renal pelvis KDSM and neoplasm (one transitionalcell carcinoma and one squamous-cell carcinoma). They were unable to provide histologic evidence, however, that the KDSM was involved in the tumor.

The presenting clinical symptoms of renal KDSM are generally nonspecific. Most commonly, patients present with flank pain (as did both of our patients), which Hertle and Androulakakis ${ }^{1}$ found in their review to be present in more than $70 \%$ of cases. Other symptoms include dysuria, pyuria, and hematuria, although none is as frequent as flank pain. ${ }^{1,3}$ The sign that is likely pathognomonic of KDSM is self-reported passage of soft gray flakes or chalky stones in the urine, giving it a turbid appearance. Both of our patients reported this sign, which has been estimated to be present in $43 \%$ of cases, ${ }^{1}$ although the actual incidence is likely much greater, because patients may not be asked specifically about this finding.

Renal KDSM has most commonly been detected as filling defects on intravenous urography or retrograde pyelography, ${ }^{1}$ because most reports of KDSM predate CT. More recent descriptions are of CT findings similar to those of our patients. 4,7,10 The general appearance of KDSM as a spaceoccupying mass within the kidney prompts consideration of malignancy.

The etiology of KDSM is in dispute, with four theories being proposed. 1,3,6 The first is that KDSM represents a reactive epithelial response to chronic irritation. Hertle and Androulakakis $^{1}$ found in their review of KDSM that 48 of 80 cases were associated with urinary tract infection, urolithiasis, tuberculosis, or hydronephrosis. Additional support is provided by the experimental findings of Plumpton and Morales, ${ }^{11}$ who demonstrated that keratinized squamous metaplasia occurs in a rat model with bladder extrophy. Both
A

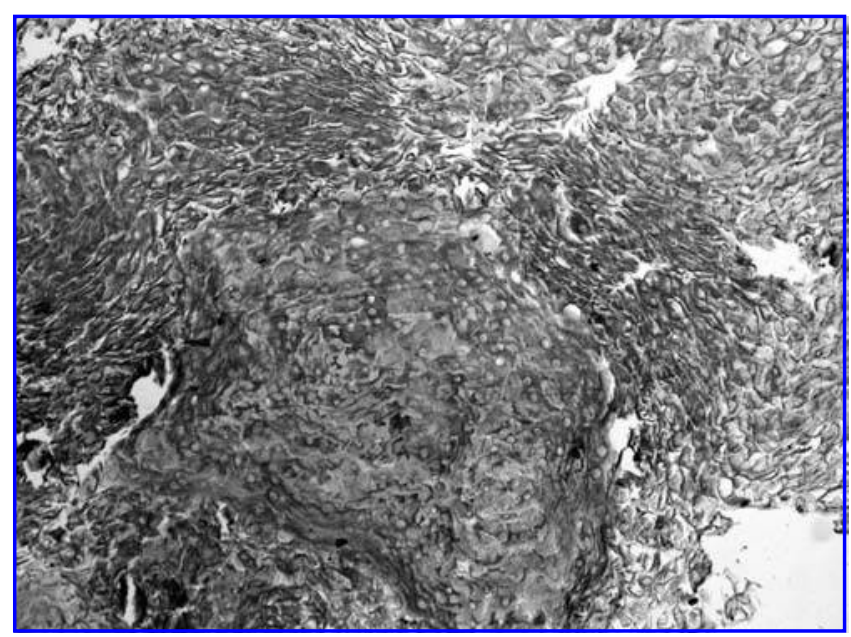

B

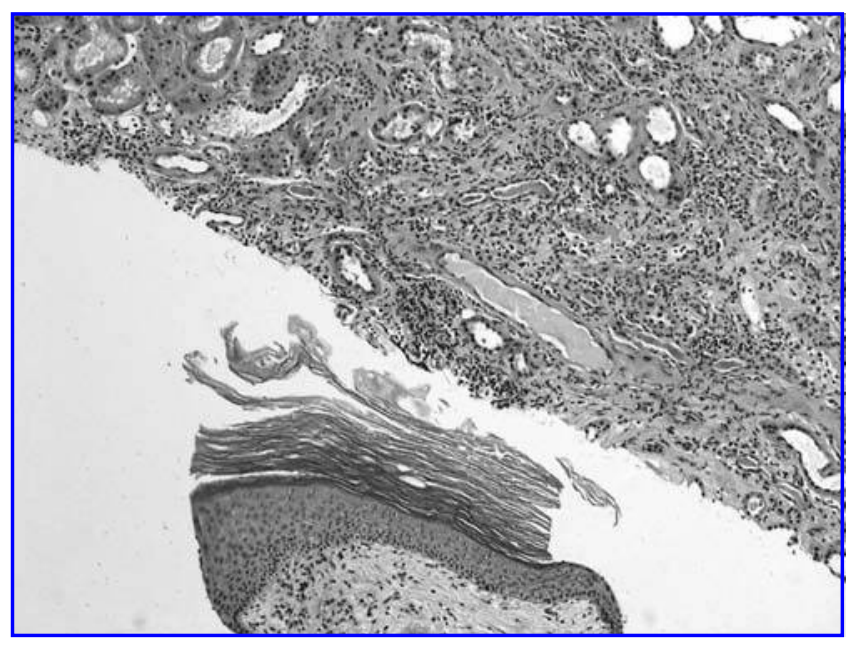

FIG. 3. (A) Cell block preparation of the washings obtained at ureteroscopy demonstrate exuberant acellular keratin debris. (B) Subsequent percutaneous core biopsy of the cystic left renal mass from the same patient confirms the presence of small fragment of benign keratinizing squamous epithelium with numerous acellular keratin materials. Separate larger fragment of renal parenchyma, seen in the upper field of the image, demonstrates interstitial inflammation and fibrosis. 
A

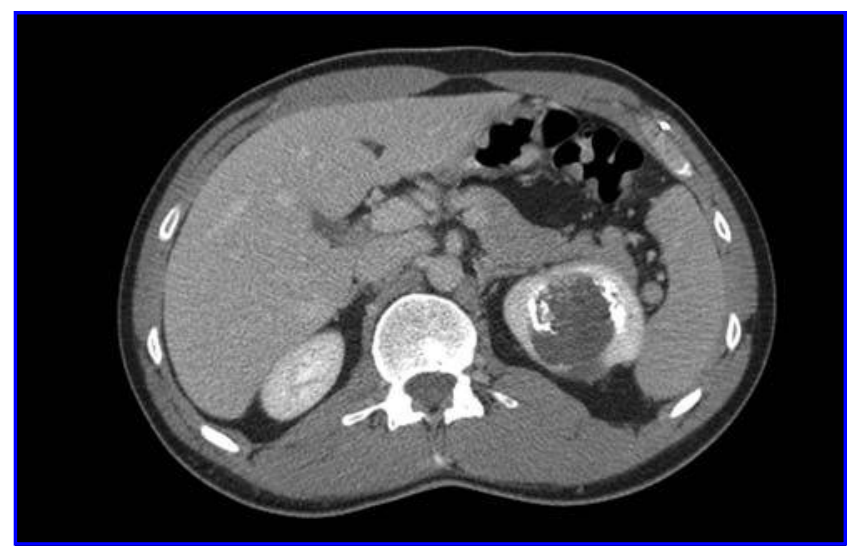

C

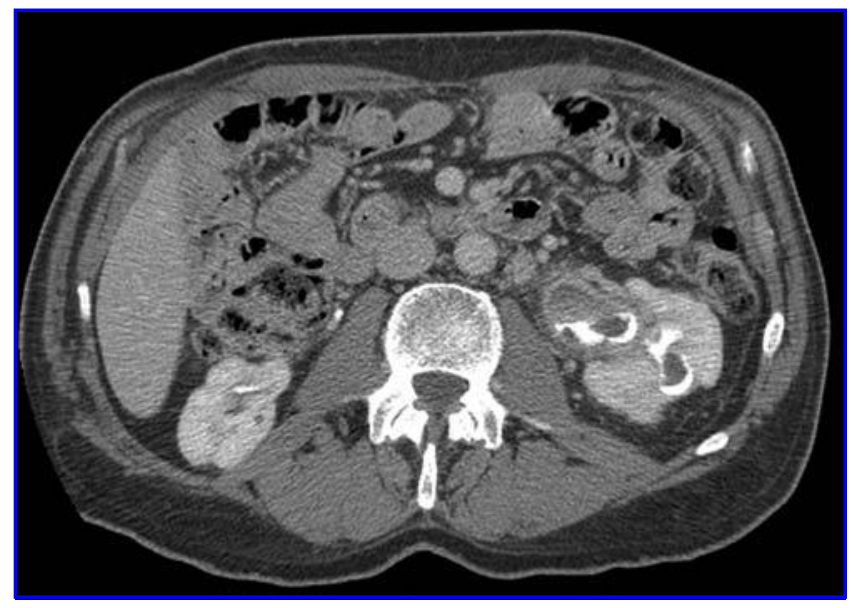

B

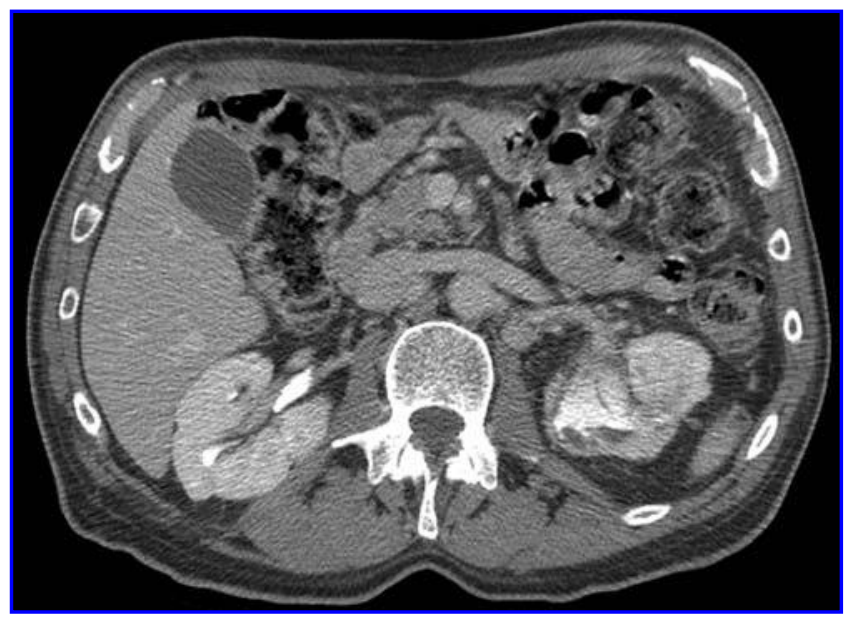

D

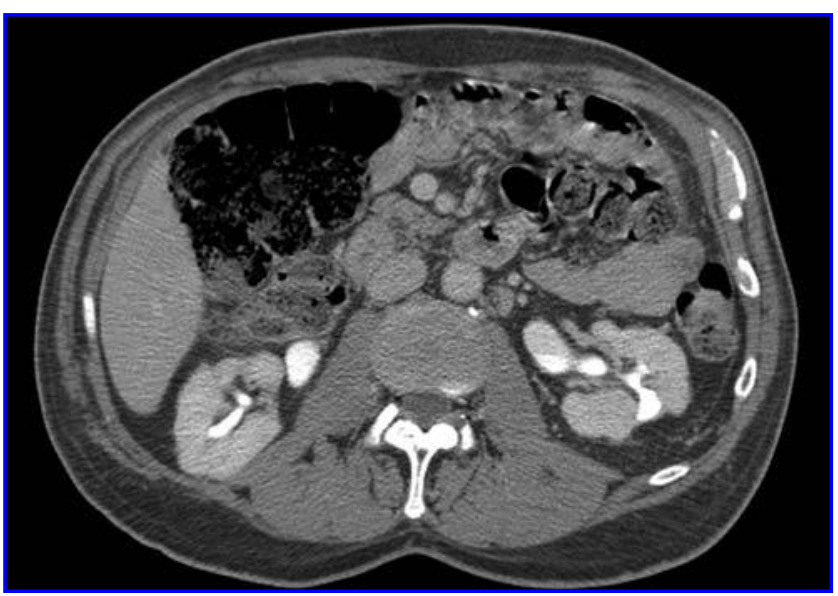

FIG. 4. (A) CT shows that the cystic mass, previously $5.0 \times 4.1 \mathrm{~cm}$, has grown to $5.8 \times 4.5 \mathrm{~cm} 42 \mathrm{months}$ later but is otherwise unchanged in appearance. (B) CT demonstrates that the cystic mass remains $3.5 \times 2.9 \mathrm{~cm}$, but now contains contrast material mixed with debris. (C) Multiple filling defects in renal pelvis and middle calyx can be seen on CT. (D) Same area as demonstrated in $4 \mathrm{C}$, from original imaging, revealing normal collecting system.

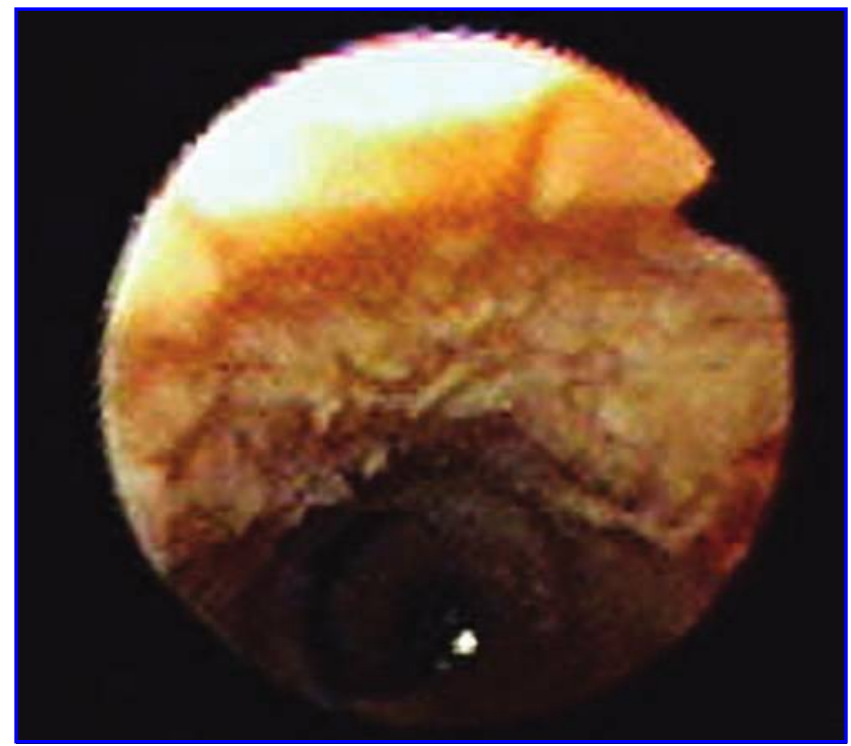

FIG. 5. Ureteroscopic view of inflamed urothelial lining of the renal pelvis. of our patients had potential upper tract irritants-a history of nephrolithiasis in one and a history of urinary tract infections plus an asymptomatic renal calculus in the other.

Second, KDSM could be secondary to vitamin A deficiency. Liang and associates ${ }^{12}$ showed that keratinizing squamous metaplasia developed in urothelial epithelium in mice fed a vitamin A deficient diet. There is also a case report in which KDSM was treated solely with vitamin A supplementation, with reported stabilization of the lesion. ${ }^{13}$

A third theory is that KDSM represents a spontaneous epithelial transformation, which would explain why renal KDSM does occur in the apparent absence of irritants. Finally, the fourth theory is that KDSM represents a congenital anomaly whereby abnormal epithelial cells from the ectoderm embryologically contaminate the primitive Wolffian duct. $^{2,3}$

Traditionally, KDSM has been managed aggressively, often with nephroureterectomy. The justifications for this approach include consideration of malignancy (if the diagnosis is in doubt), concern about malignant transformation of KDSM, and a desire to prevent recurrence by removing entirely the metaplastic epithelium. ${ }^{14}$ 
It appears very unlikely that KDSM is a premalignant lesion, but the question of recurrence is less clear. Gale and Kerr $^{15}$ managed a case of renal KDSM by surgically removing the keratinaceous debris, but there was recurrence 1 year later, and ultimately nephrectomy was performed. Conversely, Zipkin and colleagues ${ }^{5}$ reported a case of surgical removal of the debris alone without recurrence for 25 months, and Taguchi and coworkers ${ }^{9}$ described endoscopic removal of debris in one case without recurrence for 7 years.

We did not attempt to endoscopically remove the debris in either of our patients. In the younger patient, the lesion has grown slightly, but we continue surveillance, because he remains asymptomatic, and the character of the cystic mass has not changed. In the older man, we may have altered the natural history of the lesion with our ureteroscopic manipulation, which apparently increased the efflux of the debris from the source to the point that debris is now seen radiographically in previously normal parts of the collecting system (Fig. 4C).

\section{Conclusion}

The unique aspect of our two cases of KDSM is the nephron-sparing management with a combination of diagnostic ureteroscopy and imaging surveillance. The duration of follow-up without adverse events suggests that this approach will be successful, and that the finding of renal KDSM is not necessarily an indication for extirpative surgery.

Considering the relative paucity of data on KDSM in the literature and the uncertainty over the natural history of the disease process, it is our recommendation that annual imaging be performed. Clinical judgment based on symptoms and the results of imaging can then be used to determine whether repeated ureteroscopy or more invasive treatment is warranted.

\section{Disclosure Statement}

No competing financial interests exist.

\section{References}

1. Hertle L, Androulakakis P. Keratinizing desquamative squamous metaplasia of the upper urinary tract: Leukoplakiacholesteatoma. J Urol 1982;127:63-635.

2. Taylor WN. Leukoplakia of the kidney pelvis and ureter. Am J Surg 1936;32:335.

3. Reece RW, Koontz WW Jr. Leukoplakia of the urinary tract: A review. I Urol 1975;114:165-171.
4. Boswell PD, Fugitt B, Kane CJ. Keratinizing desquamative squamous metaplasia of the kidney mimicking transitional cell carcinoma. Urology 1998;52:512-513.

5. Zipkin JW, Spreen SA, Evans AT. Hyperkeratotic squamous epithelium of pelvis and ureter in solitary kidney. Urology 1985;25:625-626.

6. Dénes FT. Keratinizing squamous metaplasia of the upper urinary tract. Int Urol Nephrol 1984;16:23-28.

7. Lima DX, Rabelo EA, Salles PG. Cholesteatoma of the upper urinary tract. Int Braz J Urol 2004;30:494-495.

8. Angulo JC, Santana A, Sanchez-Chapado M. Bilateral keratinizing desquamative squamous metaplasia of the upper urinary tract. J Urol 1997;158:1908-1909.

9. Taguchi Y, Kotha V, Tomka B, Seemayer T. Conserving nephrons in cholesteatoma. J Urol 1980;123:258-260.

10. König J, Pannek J, Kickuth R, Noldus J. Late recurrence of renal cholesteatoma after 15 years. Urology 2004;64:808-809.

11. Plumpton K, Morales A. Experimental induction of urothelial metaplasia. Urology 1974;3:651-653.

12. Liang FX, Bosland MC, Huang $\mathrm{H}$, et al. Cellular basis of urothelial squamous metaplasia: Roles of lineage heterogeneity and cell replacement. J Cell Biol 2005;171:835-844.

13. Lupovitch A, Domzalski H, Tippins R. Cholesteatoma of the renal pelvis: A case with long-term followup. J Urol 1988;140:360-361.

14. Neerhut G, Politis G, Alpert L, Griffith DP. Cholesteatoma of the renal pelvis: Endoscopic management. I Urol 1988;139:1032-1034.

15. Gale GL, Kerr WK. Cholesteatoma of the urinary tract. J Urol 1970;104:71-72.

Address reprint requests to:

J. Stuart Wolf, Jr., MD

University of Michigan

Department of Urology

1500 East Medical Center Drive, TC 3875

Ann Arbor, MI 48109

E-mail: wolfs@umich.edu

$$
\begin{aligned}
& \text { Abbreviations Used } \\
& \mathrm{CT}= \text { computed tomography } \\
& \mathrm{KDSM}= \begin{array}{l}
\text { keratinizing desquamative squamous } \\
\text { metaplasia }
\end{array}
\end{aligned}
$$



This article has been cited by:

1. Anjana Ganeshappa , Amy Krambeck, David J. Grignon, James E. Lingeman . 2009. Endoscopic Management of Keratinizing Desquamative Squamous Metaplasia of the Upper Tract: A Case Report and Review of the LiteratureEndoscopic Management of Keratinizing Desquamative Squamous Metaplasia of the Upper Tract: A Case Report and Review of the Literature. Journal of Endourology 23:8, 1277-1279. [Abstract] [PDF] [PDF Plus] 